\title{
Teaching Supply Chain Management: Mixed vs. Pure Strategies in Simulation Based Training
}

\author{
Avinoam Tzimerman, Yale Herer, and Avraham Shtub \\ Technion, Israel \\ \{Avinoamt, yale, shtub\}@ie.technion.ac.il
}

\begin{abstract}
We investigate two inter-entity strategies for using SBT by teams-a competitive strategy and a cooperative strategy within the supply chain management domain. We examine how a combination of these inter-entity strategies would work at situations wherein both are used, one following the other. The goal is to investigate from a pedagogical and practical perspective which strategy is the most effective using SBT techniques for business educationpure strategy (strictly cooperative/competitive) or mixed (a combination of both). The results indicate that it is better to teach with mixed strategies when using SBT by teams, than to teach only one (pure) strategy. Moreover, if both strategies are used, then the order of teaching is significant. It is better to start with a competitive strategy and then move to a cooperative strategy rather than the reverse.
\end{abstract}

Keywords: Management Education, Simulation Based Training, Competitive, Cooperative.

\section{Introduction}

Over the past decade, there has been an enormous growth and development in business education. New programs have emerged while existing ones have been extended and enriched with upgraded content. These educational programs include courses that are taught using various pedagogical methods that include frontal lectures, books, recorded presentations, seminars, group learning, etc. by virtue of being confined to a classroom environment traditional methods have struggled to transform expert intuition and theoretical knowledge into practical experience. An emulation of a real physical environment is one way to aid in this transformation. However, efficiently and effectively providing this emulation is an important challenge of management education which has yet to be answered $[1,2]$. Nonetheless, over the past decade spectacular advances in infrastructure technologies that enable the use of modern tools to enhance learning have been proposed.

One tool that has been extensively investigated is computerized automated simulators. Simulation creates an artificial environment that reflects and illustrates real-life experiences [3]. Of particular interest to us is the use of simulation for knowledge 
transfer. This process is typified by the knowledge a trainee gains during his experience with the artificial environment (simulation) and the subsequent implementation of the knowledge. One characteristic of an efficient simulation tool is its ability to facilitate learning that can be transferred to a real-life environment [4]. In the context of education, using simulation as a teaching methodology is commonly referred to in the literature as Simulation Based Training - SBT.

SBT for business education provides several advantages over traditional techniques, such as supplying hands-on practice and allowing for the development of skills at a faster pace [5]. Salas et al. delineated seven basic stages of SBT development in business education [6]. Other researchers provided some practical guidelines as to how best to implement and use SBT in business education-e.g., offer detailed focused feedback, and directly measure outcomes [5]. Some researchers [5, 7] asserted that SBT can be an efficient tool for business education, but only when the gap between the trainee's a priori knowledge and the difficulty of the simulation exercise are matched. Inasmuch as SBT is important for current and future education, it is essential to identify the circumstances under which SBT is most effective.

In this paper, we investigate two inter-entity strategies for using SBT by teams-a competitive strategy and a cooperative strategy. Research has attempted to determine which strategy is better for training teams $[8,9,10,11]$. Sherman's study [11] showed no significant differences in cooperative and competitive learning. A recent study on these SBT inter-entity strategies was carried out within the project management domain [12] and indicated that cooperative strategies yield better results in the overall outcome. There are pros and cons for each strategy [11], yet the cooperative one is considered to be more effective $[12,13]$. Students who were trained using a cooperative strategy, which is better for long term relationships [14], tend to employ this strategy in their professional life. The parties involved are usually more satisfied, and as a result, the overall business profit rises [15]. To the best of our knowledge, no study has examined how a combination of these inter-entity strategies would work. That is to say, research has looked at the results of using one or the other strategy but not at situations wherein both are used, one following the other. This is a lacuna that we come to fill in. Unlike past research that focused on finding the best strategy we look at a combination of the two strategies. We investigate this issue within the supply chain management domain. The goal of this paper is to investigate from a pedagogical and practical perspective which strategy is the most effective using SBT techniques for business education-pure strategy (strictly cooperative/competitive) or mixed (a combination of both).

\section{Experiment Research Design}

In this section we present our experimental design, starting with our hypothesis and culminating in a detailed experimental design. In Section 3 we report on the results of the experiment and analyze the data.

\subsection{Hypothesis}

Different parties comprise the supply chain, each desiring to meet their own goals. In this pursuit they can adopt omnifarious inter-entity strategies, including competitive 
and cooperative ones, in regard to the interrelationships with their colleagues. When students are being taught about the interdependence among the supply chain parties, the teacher can choose to teach using one or both of these inter-entity strategies. Our experiment tests the following hypotheses in regard to cooperative and competitive inter-entity strategies in using SBT by teams:

Hypothesis 1: Using a mixed strategy (cooperative and competitive) achieves better learning outcomes than using a pure (single) strategy.

Hypothesis 2: When using a mixed strategy, the order of the two strategies affects the learning outcomes.

\subsection{Experiment Design}

To examine these pedagogical hypotheses we use a new advanced SBT computerized simulation environment. The software, Supply Chain Simulator (SCS), is a web based computer simulation application developed at the Technion. It is designed as an educational platform to enable students to gain better understanding and experience in managing supply chains. SCS is based on the following principles: Ease of use, Scenario based training, Flexibility, and Supportive data.

Two classes (I and II) of freshman undergraduates in the Industrial Engineering and Management Faculty of the Technion participated in the experiment. Class I consisted of 134 students and Class II consisted of 121 students. None of the students had theoretical or formal training with supply chain management. Prior to their first task using SCS the students received an introduction to supply chain management and SCS. The introduction included an oral presentation and live demonstration of how to use SCS, instructions on how to run scenarios and an explanation of the performance measure used (i.e., profit) and how it is calculated. The students were motivated to pursue the highest profit possible as this performance measure determined their grade in each task. The students ran four scenarios, two of them were individual tasks (the first and fourth scenarios) and the other two were run in pairs. The nature of the four scenarios was as follows.

Scenario 1: Managing a supply chain with an intermediate level of difficulty. The supply chain scenario comprised seven facilities (two manufacturers, two warehouses and three retailers). In the beginning of each period the students had to make several managerial decisions such as the quantity to produce at each manufacturing facility, the order quantity of each warehouse and retailer, and the supply priority in case of a conflict between external demand (customers) and internal demand (orders from another facility within the supply chain). The students' goal was to maximize the overall profit over twelve time periods. Each student had ten trials and the best performance was recorded. The purpose of this scenario, as far as this research is concerned, was to expose the students to the simulator and allow them to practice so that all groups of students would be aligned in their ability to operate the simulator.

Scenarios 2 and 3: For these scenarios (2 and 3) each class (I and II) was divided into teams of two students. Additional segmentation was done as the teams were divided randomly into two groups (A and B). The teams and the groups remained unchanged. Each scenario included a supply chain with an advanced level of difficulty that needed to be managed by the team. Each supply chain scenario comprised several 
facilities (manufacturers, warehouses and retailers). One student was in charge of the "manufacturing division," which included the manufacturers and the warehouses, and the second student was in charge of the "marketing division," which included the retailers. Each student had an individual goal to maximize his own facilities' profit over twelve time periods; nonetheless the students had to work together as a team to manage the same scenario.

The nature of the work relationship between the team members was sometimes competitive or cooperative. The different inter-entity strategies were realized in the way the students were graded. Every team had twenty trials for each scenario. In the competitive strategy, the students had to decide by themselves which trial was the one used as their grade. They had to choose the same trial for both team members even though this trial would not necessarily represent the supply chain's best overall performance. The students in the same team were nonetheless individually graded according to their individual performance, regardless of the overall team performance. Clearly, the two students on each team were at odds with each other since each team member wanted to choose the trial that was best for him and this was generally not the best for his partner. When the team members were unable to agree upon a specific trial, the average performance over the last ten trials was used as the grade.

In contrast, in the cooperative strategy the students did not have to decide which trial would be considered for their grade; the trial that produced the maximum overall profit for the entire supply chain was used. The students in the same team were still individually graded according to their individual performance.

The assignment of the teams is as follows. In Class I, the teams in Group A were assigned a competitive strategy for Scenario 2 and a cooperative strategy for Scenario 3. The assignment to Group B was reversed: the teams used a cooperative strategy in Scenario 2 and a competitive strategy in Scenario 3. Both scenarios were actually the same though the students were unaware of this fact (the facilities of the supply chain were organized differently on the map). Consequently, the potential performance (overall profit) of each exercise was the same.

In Class II, the teams in the groups were always assigned the same strategy. Teams in Group A were assigned the competitive strategy and teams in Group B were assigned the cooperative strategy.

Scenario 4: An individual task similar to the first one. The scenario had an advanced level of difficulty, requiring each student first to design an efficient supply chain using predetermined facilities and then to manage it. The goal was the same as in Scenarios 1 - 3, i.e. to maximize the overall profit over twelve time periods. The goal of the experiment was to measure each student's individual ability after they had learned and gained experience. In addition, this scenario enabled us to compare the two groups' performances. Moreover, Scenario 4 was exactly the same for the two classes, thus enabling us to compare the two classes' performances after handling Scenarios $1-3$.

\section{Experiment Results and Analysis}

For Class I we had 66 teams in Scenarios 2 and 3, 32 teams in Group A and 34 teams in Group B. In addition, we collected 120 results of individual students in Scenario 1 and 127 results of individual students in Scenario 4. For Class II we collected the 
results of 61 teams in Scenarios 2 and 3, 30 teams in the 'Comp-Comp' group and 31 teams in the 'Coop-Coop' group. In addition, we collected 111 results of individual students in Scenario 1 and 112 results of individual students in Scenario 4. The reduction in the number of students participating in the various scenarios was caused by students dropping the course. Moreover, we believe that their reasons for not continuing in the course were unrelated to the simulator. We note that the number of students dropping the course was no greater than normal. In addition, in each class there were 2-3 outliers observations that were removed. These outliers were students that achieved very law score in one of the scenarios, usually that occurred when they started the scenario but abandoned it in the middle.

Whereas one may feel that a simple t-test is suitable to check our hypotheses, we found this not to be the case. The main reason is that the within-group variance masks the differences between groups. This can be understood by the bivariate fit analysis of the performances of both classes. When analyzing Class I and II results, a strong correlation between the teams and their performance was revealed (Class I p.value $<0.002$, Class II p.value < 0.001). Fig. 1 present the bivariate fit test results of the correlation between the teams and their performance in Scenarios 1 and 4 in Class I. Class II demonstrates a similar behavior. The teams tended to show either high or low performance regardless of strategy. Due to the correlation findings and in order to reduce the withingroup variance, in all our statistical tests below we used ANCOVA to test the significance of differences between the group means of the students' performance in Scenario 4 (dependent variable) using students' performance Scenario 1 as a covariate.

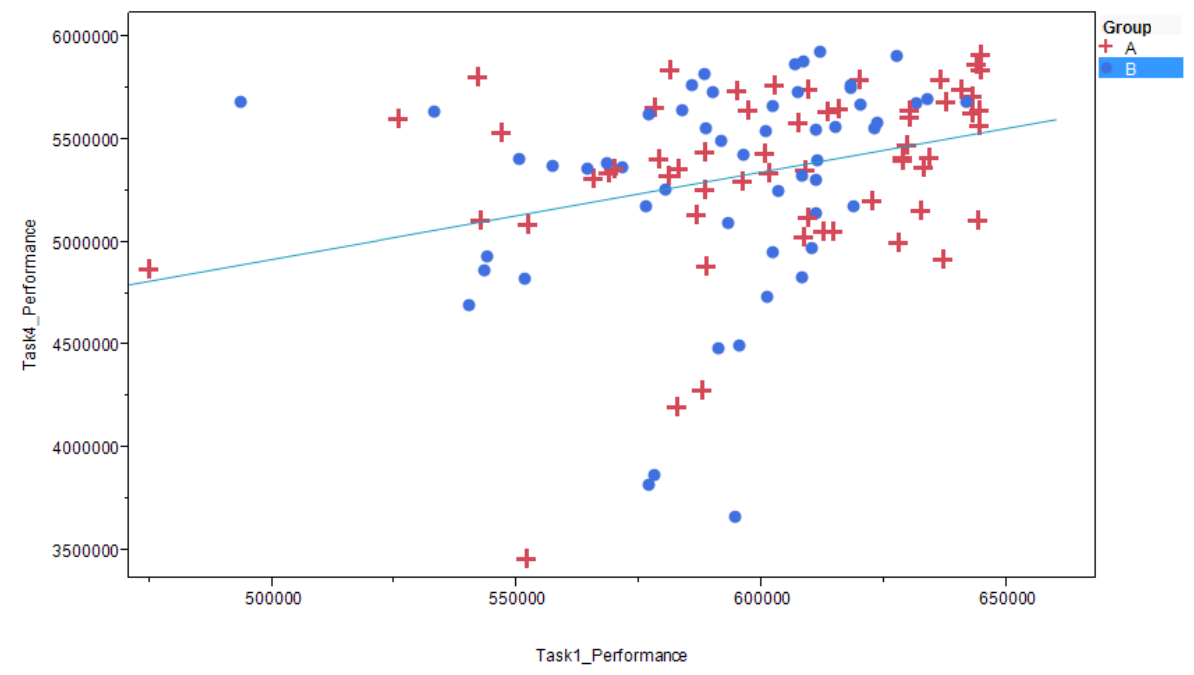

Fig. 1. Bivariate fit graph of Class I Scenarios 1 vs. 4

\subsection{Hypothesis 1}

In order to test whether a mixed strategy achieves better learning outcomes than using a pure strategy, we checked whether there is a significance difference in the performance 
of Class I vs. Class II in Scenario 4. We used ANCOVA to test the significance of differences between the means of the students' performance in Scenario 4 (dependent variable). Due to the correlation findings and in order to reduce the within-group variance, we used students' performance in Scenario 1 as a covariate. The results indicate that the performance in Scenario 4 is dependent on the Class. The significance of the model has a $\mathrm{p}$ value $<0.001$ and the significance of the independent variable (class) has also a $\mathrm{p}$ value $<0.001$.

\subsection{Hypothesis 2}

In order to test whether the order of the two strategies is significant when using a mixed strategy, we checked whether there is a significant difference in the performances in Scenario 4 between the groups of Class I. We used ANCOVA to test the significance of differences between the mean performances of the two students groups in Scenario 4 (dependent variable). Due to the correlation findings and in order to reduce the within-group variance, we again used the students' performance in Scenario 1 as a covariate. The results indicate that the performance in Scenario 4 is dependent on the Group when correcting for the groups' performance in Scenario 1. The significance of the model has a $\mathrm{p}$ value $<0.001$ and the significance of the independent variable (Group) has a $\mathrm{p}$ value of 0.007 .

\section{Discussion and Conclusions}

This research focused on team learning using SBT techniques in the business management education domain. We utilized a simulator to manage a supply chain. Two different strategies in were investigated-cooperative and competitive-trying to determine whether a pure strategy or a mixed one is the most effective when using SBT. This study, adopting statistical models to assess the effect of the variables on the students' performance, showed significant results. These results indicate that it better to teach with mixed strategies when using SBT by teams, than to teach only one (pure) strategy. Moreover, if both strategies are used, then the order of teaching is significant. It is better to start with a competitive strategy and then move to a cooperative strategy rather than the reverse.

The students from Class I in Scenario 4 outperformed the students from Class II in this scenario, thus it supports our first hypothesis that it is better to teach both strategies than only one. A possible explanation to the observed phenomena is that teaching both strategies when using SBT by teams, exposes the students to a broader experience than students who are being taught by only one strategy. This experience enriches the learning process thus it is more efficient.

It seems that teams of Class I that started with a competitive strategy and then used a cooperative strategy learned from this process. The learning was expressed by their better performance of the teams when using a cooperative strategy than a competitive one; i.e., the second round performance was better than the first one. In contrast, teams that started with a cooperative strategy and then used a competitive one did not learn enough from the first round in order to improve their performance in the second 
round. This observation is supported by the zero difference in the performance of the first and second rounds for the teams using a cooperative and then a competitive strategy. That being the case, allegedly learning from Scenario 2 to Scenario 3 in Class I did not occur (on average) for a team that started with a cooperative strategy.

An additional finding that strengthens the assertion that it is better to start with competitive strategy and then move to cooperative is the students' performance in Scenario 4, which enabled us to compare all the participants' performance. In Scenario 4 the students of Class I that started with the competitive task significantly outperformed the students who started with the cooperative task.

A potential explanation to the differences in the performances of the two groups in Class I is that a competitive exercise causes the team to experience the negative effects of competition and thus during the next session, the team members will appreciate the opportunity to cooperate. Accordingly, one may conclude that learning occurs when the student starts from the "negative example" (competitive), and not the other way around. That is to say that a "negative example" is a better way to induce learning.

Another possible explanation is that a team that started with a cooperative strategy has already internalized the benefits of cooperation. Thus when the team faced a competitive strategy (scenario 3), its members did not act according to the competitive instructions, but continued to act as if they were in a cooperative exercise. It is possible that trying to teach a competitive strategy after a cooperative strategy has already been taught is not effective, as the participants do not follow the competitive strategy instructions but prefer to act as if using a cooperative strategy. The effect here was intensified because the competitive teams were not motivated to act competitively - there was no penalty for them if they did not do so.

A technical explanation for the above findings may perhaps be that a competitive exercise could be more effectively handled from the simulator point of view. It forces each member of the team to deal with the exercise (each team member must take care of himself if he wants to succeed, whereas in a cooperative exercise, each team member knows he can count on his partner).

After considering the above explanations, we propose some guidelines on how to use SBT for team training:

- Teach using both cooperative and competitive strategies (and not just one of them) when using SBT by teams.

- When training teams and trying to encourage a certain behavior and eliminate another behavior considered to be negative, it is better for trainees to first gain experience with the negative behavior and its repercussions; only afterwards should they be exposed to the desired behavior and its benefits because in the latter situation the differences are more obvious and the learning process is intensified.

- Even if teams are being trained in the reverse order (i.e., first the desired behavior and only afterwards the negative one), then make sure that the teams follow the instructions and actually act upon the negative behavior, they should be given motivation to do so.

- Even though the team acts as one unit, in order to ensure that each member participates fully in the exercise and personally gains from it, each should be given personal responsibility and a separate grade. This minimizes the free rider phenomenon. 


\section{References}

1. Gunes, M., Baba, A.F.: Educational tool for design and implementation of an autonomous mobile robot. International Journal of Engineering Education 25(2), 239-249 (2009)

2. Doulgeri, Z., Zikos, N.: Development, integration and evaluation of a web-based virtual robot task simulator in the teaching of robotics. International Journal of Engineering Education 25(2), 261-271 (2009)

3. Bell, B.S., Kanar, A.M., Kozlowski, S.W.: Current issues and future directions in simulation-based training in North America. International Journal of Human Resource Management 19, 1416-1434 (2008)

4. Thompson, B., Bergus, G.: Simulation in medical education, report of the task force on simulators in education. In: CCOM Medical Education Committee (June 2006)

5. Salas, E., Wildman, J.L., Piccolo, R.F.: Using simulation-based training to enhance management education, . The Academy of Management Learning and Education (AMLE) 8(4), 559-573 (2009)

6. Salas, E., Wilson, K.A., Burke, C.S., Priest, H.A.: Using simulation-based training to improve patient safety: What does it take? Journal on Quality and Patient Safety 31(7), 363$371(2005)$

7. Shtub, A., Zwikael, O.: On the use of simulation based training for management education: Mind the gap as one size does not fit all, Working paper. Australian National University (2011)

8. Okebukola, P.A.: The Relative Effectiveness of Cooperative and Competitive Interaction Techniques in Strengthening Students' Performance in Science Classes. Science Education 69(4), 501-509 (1985)

9. Johnson, R.T., Johnson, D.W., Stanne, M.B.: Comparison of Computer-Assisted Cooperative, Competitive, and Individualistic Learning. American Educational Research Journal 23(3), 382-392 (1986)

10. Tjosvold, D.: Cooperative and Competitive Goal Approach to Conflict: Accomplishments and Challenges. Applied Psychology: An International Review 47(3), 285-342 (1998)

11. Sherman, L.W.: Cooperative Versus Competitive Educational Psychology Classrooms: A Comparative Study. Teaching and Teacher Education 2(4), 283-295 (1986)

12. Nembhard, D., Yip, K., Shtub, A.: Comparing competitive and cooperative strategies for learning project management. Journal of Engineering Education 98(2), 181-192 (2009)

13. Johnson, D.W., Maruyama, G., Johnson, R., Nelson, D., Skon, L.: Effects of cooperative, competitive, and individualistic goal structures on achievement: A meta-analysis. Psychological Bulletin 89, 47-62 (1981)

14. Su, Q., Song, Y.-T., Li, Z., Dang, J.-X.: The impact of supply chain relationship quality on cooperative strategy, . Journal of Purchasing and Supply Management 14(4), 263-272 (2008)

15. Benton, W.C., Maloni, M.: The Influence of Power Driven Buyer/Seller Relationships on Supply Chain Satisfaction. Journal of Operations Management 23(1), 1-22 (2005) 